\title{
Numerical Modeling of the Time Evolution of Super-Small-Scale Irregularities in the Near-Earth Rarefied Plasma
}

\author{
Oleg V. Mingalev, Mikhail N. Melnik, Victor S. Mingalev* \\ Polar Geophysical Institute, Russian Academy of Sciences, Murmansk, Russia \\ Email: ${ }^{*}$ mingalev@pgia.ru
}

Received 3 January 2015; accepted 25 January 2015; published 28 January 2015

Copyright (C) 2015 by authors and Scientific Research Publishing Inc.

This work is licensed under the Creative Commons Attribution International License (CC BY). http://creativecommons.org/licenses/by/4.0/

c) (7) Open Access

\begin{abstract}
The time evolution of the magnetic field aligned super-small-scale irregularities in the concentration of charged particles, existing in the near-Earth rarefied plasma, is studied with the help of the model simulation. A new version of the two-dimensional mathematical model, developed earlier in the Polar Geophysical Institute, is utilized to investigate the temporal history of the irregularity with circular cross section, created initially in the near-Earth plasma. The utilized model is based on a numerical solution of the Vlasov-Poisson system of equations, with the Vlasov equations describing the distribution functions of charged particles and the Poisson equation governing the self-consistent electric field. The results of simulation indicate that the mobility of the positive ions ought to influence essentially on the time evolution of the super-small-scale irregularities in the concentration of charged particles, existing in the near-Earth rarefied plasma.
\end{abstract}

\section{Keywords}

Near-Earth Plasma, Super-Small-Scale Irregularities, Numerical Simulation

\section{Introduction}

Irregularities in the concentration of charged particles are often observed in the near-Earth plasma. These irregularities are a natural phenomenon in the Earth's ionosphere and magnetosphere. The irregularities have a wide range of spatial scales, ranging from thousands of kilometers to a few Debye lengths. The charged particles density increases and depletions inside irregularities can lie in the range from some tens of percentages to a few

${ }^{*}$ Corresponding author.

How to cite this paper: Mingalev, O.V., Melnik, M.N. and Mingalev, V.S. (2015) Numerical Modeling of the Time Evolution of Super-Small-Scale Irregularities in the Near-Earth Rarefied Plasma. International Journal of Geosciences, 6, 67-78. 
portions. In the ionosphere, the well-known equatorial anomaly at F-layer altitudes is the example of large-scale irregularities [1]. The main ionospheric trough, observed at subauroral latitudes, is another example of largescale irregularities in the ionospheric F-layer [2]. Some types of large-scale irregularities are also observed in the high-latitude ionosphere, for example, the tongue of increased values of the electron density stretched over the polar cap from the dayside to night side, polar and auroral peaks of ionization, "patches" and "blobs" of ionization and so on [3]-[6]. The F-layer ionosphere can contain middle-scale irregularities with dimensions of tens of kilometers, too [7] [8]. Short-scale irregularities, having cross-section diameters of about hundreds of meters, are also observed in the Earth's ionosphere [9]-[12]. Also, the well-known example of short-scale irregularities is a radio aurora or small-scale turbulence of the ionospheric E-layer [13]. An example of irregularities in the ionosphere is meteor plasma trails, also [14].

Not large-scale irregularities are predominately magnetic field aligned. Usually, there are three generic types of their structures: rods, wings, and sheets. Rods are isotropic in the plane perpendicular to the geomagnetic field. Wings and sheets are elongated not only along geomagnetic field but also in the perpendicular plane along a certain direction [15].

It is known that the irregularities either are naturally present or may be artificially produced as a result of active experiments in the near-Earth plasma. For example, both a large-scale irregularity and super-small-scale irregularities in the concentration of charged particles may be formed by high-power high-frequency radio waves, pumped into the ionosphere by ground based ionospheric heaters. To investigate the behavior of the artificially created irregularities in the near-Earth plasma not only the experimental and theoretical but also computational studies may be applied. The large-scale F-layer modification by powerful high frequency waves was investigated with the help of mathematical models in some studies (for example, see [16]-[29]). Also, the time evolution of the magnetic field aligned super-small-scale irregularities in the concentration of charged particles, existing in the near-Earth rarefied plasma, was studied with the help of the model simulation [30]-[33].

The purpose of the present paper is to investigate numerically the time evolution of the magnetic field aligned super-small-scale irregularities in the concentration of charged particles, existing in the near-Earth rarefied plasma, whose cross section is much less than the mean free path of particles between successive collisions and commensurable with a Debye length.

\section{Mathematical Model}

The near-Earth plasma at altitudes of the ionospheric F layer and magnetosphere is a rarefied compound consisting of electrons and positive ions in the presence of a strong, external, magnetic field. The parameters of the magnetic field aligned irregularities, studied in the present paper, have been described in details by Wong et al. [34]. According to their paper, diametrical sizes of these irregularities are several Debye lengths (no more that about 100), while the disturbances of the density of charged particles in them can reach some tens of percentages. These irregularities may be formed in the near-Earth plasma not only by natural processes but also as a result of active experiments [34].

The studied irregularities are geomagnetic field-aligned. In the vicinity of the irregularity, gradients of the plasma parameters in the longitudinal direction are supposed to be much less than those in a plane perpendicular to a magnetic field. Therefore, plasma parameters inside and beyond the irregularity may be considered as independent on the longitudinal coordinate. Hence, it is sufficient to consider a two dimensional flow of plasma in a plane perpendicular to a magnetic field line.

In the present paper, we study the magnetic field aligned irregularities, having circular cross sections, that is, the rodlike irregularities, with their diametrical sizes being much less than the mean free path of particles between successive collisions. To investigate the time evolution of the studied irregularities a two-dimensional mathematical model, developed earlier in the Polar Geophysical Institute, is utilized. In this model, kinetic processes in the plasma are simulated by using the Vlasov-Poisson system of equations, with the Vlasov equations describing the distribution functions of charged particles and the Poisson equation governing the self-consistent electric field. The Vlasov equations are numerically solved applying a macroparticle method. The Poisson equation is solved using a finite-difference method. The utilized system of equations has been earlier considered, for example, in the studies by Hockney and Eastwood [35] and Birdsall and Langdon [36]. This system may be written as follows: 


$$
\begin{gathered}
\frac{\partial f_{a}}{\partial t}+\left(\mathbf{v}, \frac{\partial f_{a}}{\partial \mathbf{x}}\right)+\frac{q_{a}}{m_{a}}\left(\mathbf{E}+\left[\mathbf{v} \times \mathbf{B}_{0}\right], \frac{\partial f_{a}}{\partial \mathbf{v}}\right)=0, \quad a=i, e \\
\Delta \varphi(\mathbf{x}, t)=-\frac{1}{\varepsilon_{0}} \rho(\mathbf{x}, t) \\
\mathbf{E}(\mathbf{x}, t)=-\nabla \varphi(\mathbf{x}, t), \rho(\mathbf{x}, t)=e_{0}\left(n_{i}-n_{e}\right), n_{a}(\mathbf{x}, t)=\int f_{a}(t, \mathbf{x}, \mathbf{v}) d \mathbf{v},
\end{gathered}
$$

where $f_{a}(t, \mathbf{x}, \mathbf{v}), n_{a}(\mathbf{x}, t), m_{a}$, and $q_{a}$ are, respectively, the distribution function, concentration, mass, and charge of particles of type $a, \mathbf{x}$ is the space coordinate vector, $\mathbf{v}$ is the velocity, $\mathbf{B}_{0}$ is the external magnetic field, $\mathbf{E}$ is the self-consistent electric field, $\varphi(\mathbf{x}, t)$ is the electric field potential, $\rho(\mathbf{x}, t)$ is the electric charge density, $\varepsilon_{0}$ is the dielectric constant of free space, and $e_{0}$ is the proton charge. The Vlasov Equation (1), describes the behavior of the distribution function of electrons $(a=e)$ and ions $(a=i)$, whereas Poisson Equation (2), describes the self consistent electric field in the plasma. More complete details of the utilized mathematical model, developed earlier in the Polar Geophysical Institute, may be found in the studies of Mingalev et al. [31]-[33].

In the latter studies, the mathematical model has been utilized for numerical simulation of the behavior of super-small-scale rodlike and sheetlike irregularities existing in the near-Earth rarefied plasma. The time evolutions of plasma irregularities, having initial cross-section dimension commensurable with a Debye length, have been simulated during the period sufficient for the irregularities to decay completely. In these studies, a non-stationary process started from a situation when, at the initial moment, the spatial distribution of the electron concentration contained an irregularity at the center of the simulation region, with the spatial distribution of the positive ion concentration having been homogeneous in all simulation region. Thus, an electrical neutrality of the plasma was disturbed at the initial moment.

Unlike, in the present study, a non-stationary process starts from a situation when, at the initial moment, the spatial distribution of the electron concentration coincides with the spatial distribution of the positive ion concentration in all simulation region. Thus, the process starts from the completely electrically neutral state in all simulation region. However, the spatial distributions of the electron and positive ion concentrations may be inhomogeneous and can contain an irregularity at the center of the simulation region at the initial moment.

Moreover, in the present study, a new version of the mathematical model is applied in which positive ions can move. In the mathematical model, utilized in the studies of Mingalev et al. [31]-[33], positive ions were supposed to be immovable. This supposition was based on the fact that the mass of the positive ion is much more than the mass of the electron. Therefore, a mobility of the positive ions must be much less than a mobility of the electrons. Nevertheless, in the new version of the mathematical model, a motion of the positive ions is taken into account.

Also, in the new version of the mathematical model, some parameters of the numerical method were improved. In particular, the quantity of the grid cells was enlarged from $768 \times 768$ to $1024 \times 1024$, the average number of macro-particles in the Debye cell for the model plasma was enlarged from $2^{13}$ to $2^{15}$. A two-dimensional simulation region, lain in the plane perpendicular to the magnetic field line, is a square and its side length became equal to 128 Debye lengths of the plasma (instead of 96 Debye lengths of the plasma in the previous version of the mathematical model).

\section{Presentation and Discussion of Results}

The utilized mathematical model can describe the time evolution of the magnetic field aligned super-small-scale irregularities in the concentration of charged particles, having various parameters at the initial moment. The results of calculations to be presented in this paper were obtained for the following initial state of the irregularity. The spatial distribution of the electron concentration (equal to the positive ion concentration at the initial moment), contains a circular irregularity at the center of the simulation region. Beyond this irregularity, the electron concentration is homogeneous and coincides with the non-disturbed electron concentration, $n_{0}$, which must be given in the simulation region at the initial moment. The initially created irregularity has the cross-section diameter of $12 \lambda_{D e}^{0}$, where $\lambda_{D e}^{0}$ is the Debye length of the plasma. Inside the irregularity, homogeneity of the plasma is broken and a part of charged particles travels from the internal circle, having the diameter of $6 \lambda_{\mathrm{De}}^{0}$, 
into the external ring, surrounding the internal circle. The relative decrease of the concentration of charged particles, $\left(n_{0}-n_{e}\right) / n_{0}$, is equal to 0.1 in the internal circle at the initial moment. In other words, 10 percent of charged particles were displaced from the center of the irregularity to its periphery, with the plasma having been electrically neutral at the initial moment.

The applied mathematical model can describe the behavior of the near-Earth plasma under various conditions. The results of calculations to be presented in this paper were obtained using the input parameters of the model typical for the nocturnal ionospheric plasma at the altitude of $300 \mathrm{~km}$. At this altitude, the plasma contains only positive ions, with the oxygen ion, $\mathrm{O}^{+}$, being the bulk of ion content (99\%). At the altitude of $300 \mathrm{~km}$, the value of the non-disturbed electron concentration (equal to the positive ion concentration), $n_{0}$, is equal to $10^{11} \mathrm{~m}^{-3}$. The electron and ion temperatures are supposed to be equal to $1213 \mathrm{~K}$ and $930 \mathrm{~K}$, respectively. The bulk flow velocities of electrons and positive ions are assumed to be zero. The value of the magnetic field, $B_{0}$, is $4.4 \times 10^{-5} \mathrm{~T}$.

The above pointed out values yield the following quantities of some physically significant parameters. The electron thermal velocity, $V_{\text {Te }}$, is equal to $135.6 \mathrm{~km} / \mathrm{s}$, the ion thermal velocity, $V_{\mathrm{Ti}}$, is equal to $0.693 \mathrm{~km} / \mathrm{s}$, the equilibrium plasma frequency, $\omega_{p e}^{0}$, is $1.78 \times 10^{7} \mathrm{~s}^{-1}$. The Debye length of the plasma, $\lambda_{D e}^{0}$, defined as $\lambda_{\mathrm{De}}^{0}=V_{\text {Te }} / \omega_{\text {pe }}^{0}$, is equal to $7.6 \times 10^{-3} \mathrm{~m}$, the electron gyro radius, $R_{c e}$, is $17.5 \times 10^{-3} \mathrm{~m}$. The equilibrium period of Langmuir oscillations of electrons, $T_{p e}$, is $3.52 \times 10^{-7} \mathrm{~s}$. The period of cyclotron oscillations of electrons, $T_{c e}$, is equal to $8.12 \times 10^{-7} \mathrm{~s}$, that is, approximately a factor of 2.3 larger than the equilibrium period of Langmuir oscillations of electrons $\left(T_{c e} \approx 2.3 \times T_{p e}\right.$ ). The collision mean free path of charged particles is about $50 \mathrm{~m}$. The mean free time of electron between successive collisions with other particles, $T_{\text {free path }}$, is larger than the equilibrium period of Langmuir oscillations of electrons by a factor of about $1047\left(T_{\text {free path }} \approx 1047 T_{p e}\right)$.

We have calculated the time evolution of the distribution functions of charged particles as well as self-consisting electric field, taking the input parameters of the mathematical model typical for the nocturnal ionosphere at the level of $300 \mathrm{~km}$, for two distinct cases. The previous version of the mathematical model was utilized for the first case, whereas, the new version of the mathematical model was used for the second case.

Simulation results, obtained for the first case when positive ions were supposed to be immovable, indicate that, after initial moment, the spatial distribution of the electron concentration changes essentially while the positive ion concentration is retained practically invariable. As a consequence, after initial moment, the electrical neutrality of the plasma was broken and an electric charge arises at the center of the simulation region. The configuration of the disturbed area in the spatial distribution of the electric charge density was similar to the configuration of the disturbed area in the spatial distribution of the concentration of charged particles at the initial moment, which contained a circular irregularity at the center of the simulation region. Further calculations indicated that the changes in the spatial distribution of the electric charge density were continued. After a short period, the disturbed area in the spatial distribution of the electric charge density vanished almost completely, with the plasma having become almost electrically neutral in all simulation region. It turned out that, after a short period, the disturbed area in the spatial distribution of the electric charge density was recovered. Later, the cycle of vanishing and recovering of the disturbed area in the spatial distribution of the electric charge density was repeated again and again. In the course of time, the disturbed area in the spatial distribution of the electric charge density was enlarged, and additional rings were arisen around the position of the circular initial irregularity situated in the center of the simulation region. The spatial distribution of the normalized electric charge density, $\rho /\left(e_{0} n_{0}\right)$, calculated for some moments, are presented in left column of Figure 1. Time variations of the electric field components at two points of the simulation region and the time variation of the normalized potential energy of the plasma, filling up all simulation region, are presented in Figures 2-6 by solid lines for the first case when positive ions were supposed to be immovable. It can be seen that, for the first case, the initially created irregularity vanishes and recovers periodically, with its parameters fluctuating. The period of the fluctuations with the maximal amplitudes is close to the period of cyclotron oscillations of electrons which is approximately a factor of 2.3 larger than the equilibrium period of Langmuir oscillations of electrons.

Let us compare the results, obtained in the present paper for the first case, when the process starts from the completely electrically neutral state in all simulation region, with the results, obtained in the studies of Mingalev et al. [31]-[33], when the electric neutrality of the plasma was broken inside the irregularity at the initial moment. These results of modeling illustrate both common characteristic features and distinctions caused by different initial conditions. The time evolutions of the magnetic field aligned super-small-scale irregularities display the following common features. Excepting a short initial period, the behavior of these irregularities is qualitatively similar. The irregularities vanish and recover periodically, with its parameters fluctuating. The periods 

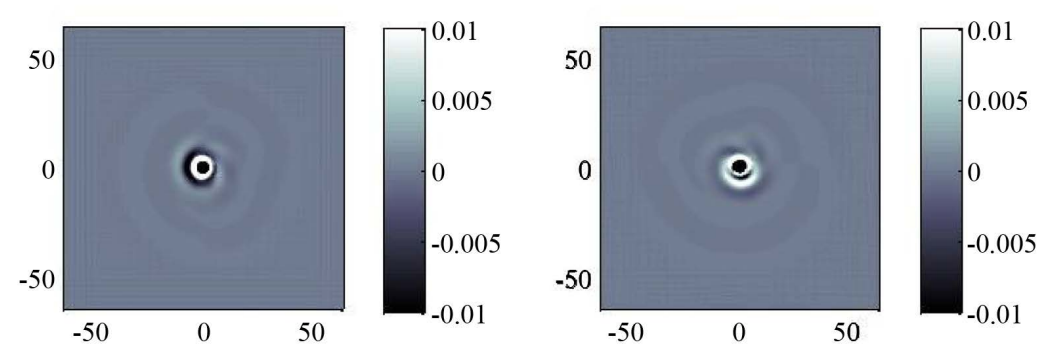

(a)
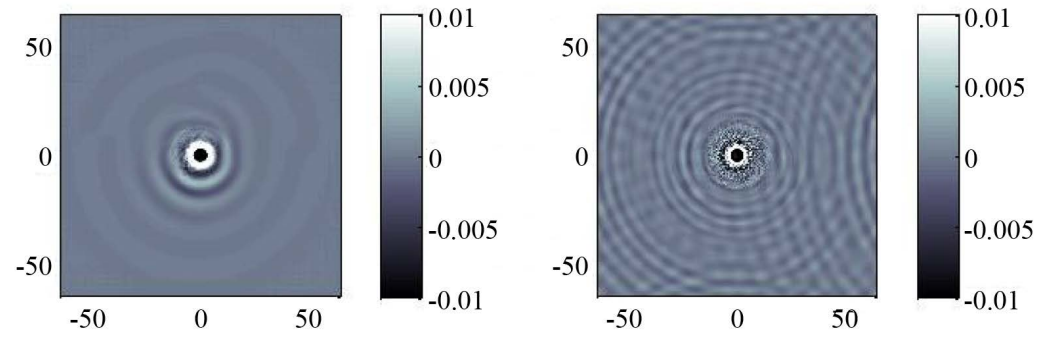

(b)
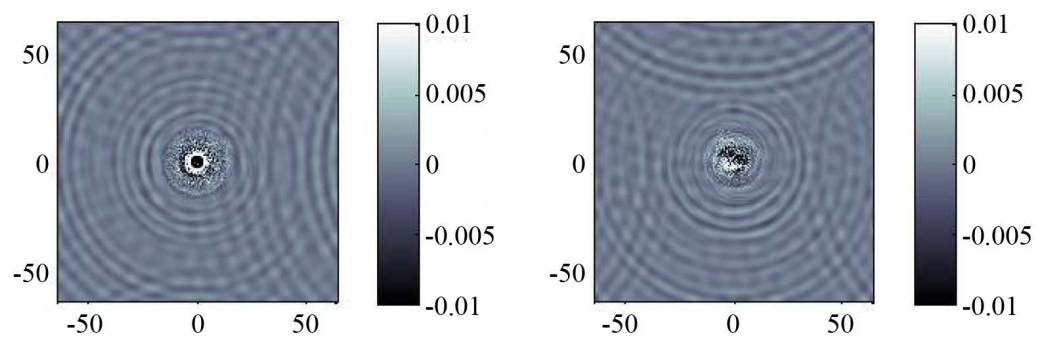

(c)

Figure 1. The calculated spatial distributions of the normalized electric charge density, $\rho /\left(e_{0} n_{0}\right)=\left(n_{i}-n_{e}\right) / n_{0}$, in the plane perpendicular to the magnetic field. The normalized distances, $\mathbf{X} / \lambda_{\text {De }}^{0}$ and $\mathbf{Y} / \lambda_{\text {De }}^{0}$, that is, the distances in units of the Debye length, $\lambda_{D e}^{0}$, from the central point of the simulation region are shown on the horizontal $(\mathbf{X})$ and vertical $(\mathbf{Y})$ axes. For the first case, when positive ions were supposed to be immovable, the results are presented in the left column, whereas, for the second case, when positive ions can move, the results are presented in the right column. The results are given for the following moments: (a) $t=4.68 T_{p e}$; (b) $t=40 T_{p e}$; (c) $t=100 T_{p e}$.

of the fluctuations are very similar. During the processes of evolutions, around the initial irregularities, additional almost symmetrical alternate rings with an excess of charge of different sign began to appear. In the course of time, these additional rings filled up all simulation region.

The time evolutions of the magnetic field aligned super-small-scale irregularities display the following distinctions caused by different initial conditions. It may be recalled that the time interval of about 35 periods of Langmuir oscillations of electrons was sufficient for the irregularity to decay completely, when the electric neutrality of the plasma was broken inside the irregularity at the initial moment [31]-[33]. Unlike, when the process starts from the completely electrically neutral state in all simulation region, the irregularity do not decay and exists during all period of calculations (100 periods of Langmuir oscillations of electrons). Such behavior takes place by reason of the presence of the irregularity in the distribution of the positive ion concentration at the center of the simulation region at the initial moment. Due to the fact that positive ions are supposed to be immovable, the irregularity in the distribution of the positive ion concentration at the center of the simulation region 


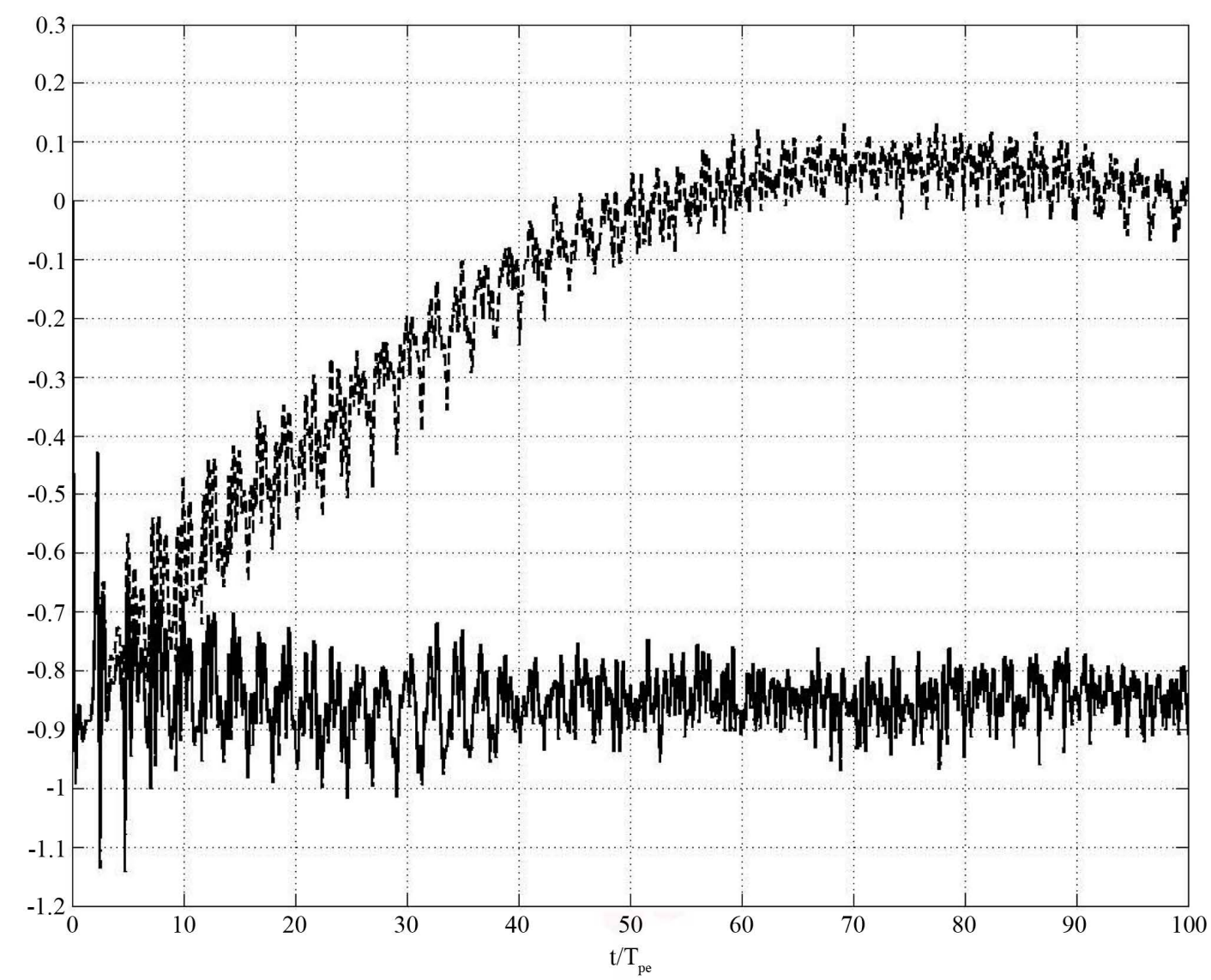

Figure 2. The time variations of one component of the electric field, namely $E_{x}$, lying in the plane perpendicular to the magnetic field, calculated at the point, displaced from the center of the simulation region in the $\boldsymbol{X}$ direction for a distance of three Debye lengths $\left(3 \lambda_{D e}^{0}\right)$. For the first case, when positive ions were supposed to be immovable, the results are shown by solid line, whereas, for the second case, when positive ions can move, the results are shown by dashed line. The electric field component is given in $\mathrm{V} / \mathrm{m}$. The normalized time, $t / T_{p e}$, that is, the time in units of the equilibrium period of Langmuir oscillations of electrons, $T_{p e}$, is shown on the abscissa.

exists during all period of calculations. As a consequence, an electrical neutrality of the plasma can not be reached because of a mobility of the electrons whose concentration deviate from the concentration of positive ions after the initial moment.

Let us consider the results, obtained in the present paper for the second case, when the process starts from the completely electrically neutral state in all simulation region and when a motion of the positive ions is taken into account. The spatial distribution of the normalized electric charge density, $\rho /\left(e_{0} n_{0}\right)$, calculated for some moments, are presented in right column of Figure 1. Time variations of the electric field components at two points of the simulation region and the time variation of the normalized potential energy of the plasma, filling up all simulation region, are presented in Figures 2-6 by dashed lines for the second case when a motion of the positive ions is taken into account. It is seen that the discussed results have some common features with the results, obtained for the first case when positive ions were supposed to be immovable. In both cases, the irregularities vanish and recover periodically, with its parameters fluctuating. The periods of the fluctuations are very similar, with the fluctuations possessing of two main periods, namely, the period of Langmuir oscillation of electrons and period of cyclotron oscillations of electrons. Furthermore, in the course of time, around the initial irregularities, additional almost symmetrical alternate rings with an excess of charge of different sign began to appear. During the processes of evolutions, these additional rings filled up all simulation region. 


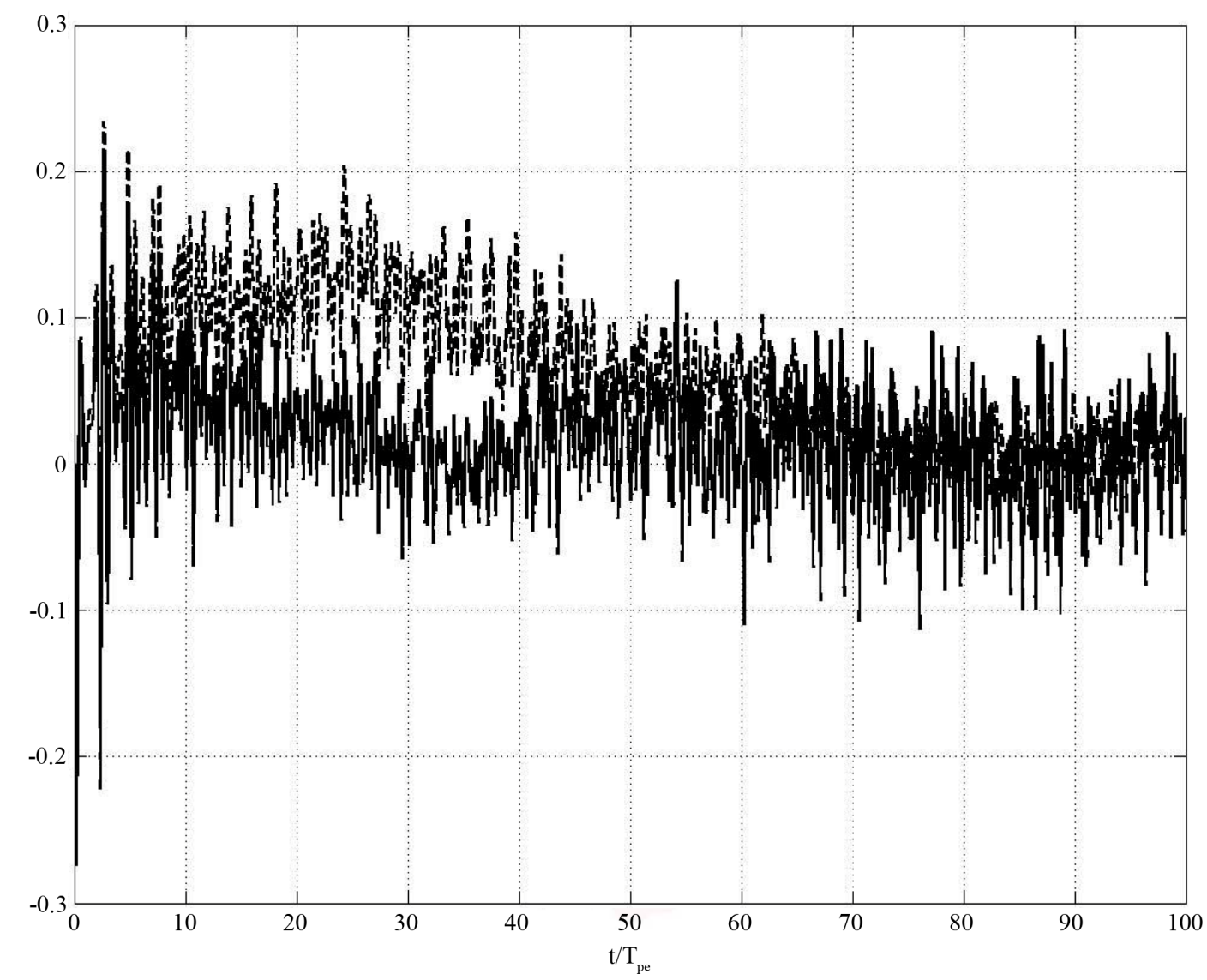

Figure 3. The time variations of one component of the electric field, namely $E_{y}$, lying in the plane perpendicular to the magnetic field, calculated at the point, displaced from the center of the simulation region in the $\boldsymbol{X}$ direction for a distance of three Debye lengths $\left(3 \lambda_{\text {De }}^{0}\right)$. For the first case, when positive ions were supposed to be immovable, the results are shown by solid line, whereas, for the second case, when positive ions can move, the results are shown by dashed line. The electric field component is given in $\mathrm{V} / \mathrm{m}$. The normalized time, $t / T_{p e}$, that is, the time in units of the equilibrium period of Langmuir oscillations of electrons, $T_{p e}$, is shown on the abscissa.

The simulation results, obtained in the present study for two different cases, have essential distinctions. In the vicinity of the center of the simulation region, for the first case, when positive ions were supposed to be immovable, the normalized electric charge density stays negative during all period of calculations, whereas, for the second case, when positive ions can move, the sign of the normalized electric charge density can change in the course of time (Figure 1). It is seen from Figure 2 that the time interval of about 50 equilibrium periods of Langmuir oscillations of electrons $\left(\approx 50 T_{p e}\right)$ is sufficient for the electric charge to change its sign in the vicinity of the center of the simulation region. Just the same time interval is required for the normalized potential energy of the plasma filling up all simulation region, $W_{p o t}(t) / W_{k i n}^{0}$, to decrease and to achieve a stable regime for the second case, when positive ions can move (Figure 6). Thus, a capacity of positive ions to move ought to influence essentially on the behavior of the magnetic field aligned super-small-scale irregularities in the concentration of charged particles, existing in the near-Earth rarefied plasma.

\section{Conclusions}

The time evolution of the magnetic field aligned super-small-scale irregularities in the concentration of charged particles, existing in the near-Earth rarefied plasma, was studied with the help of the model simulation. The twodimensional mathematical model, developed earlier in the Polar Geophysical Institute, has been improved by 


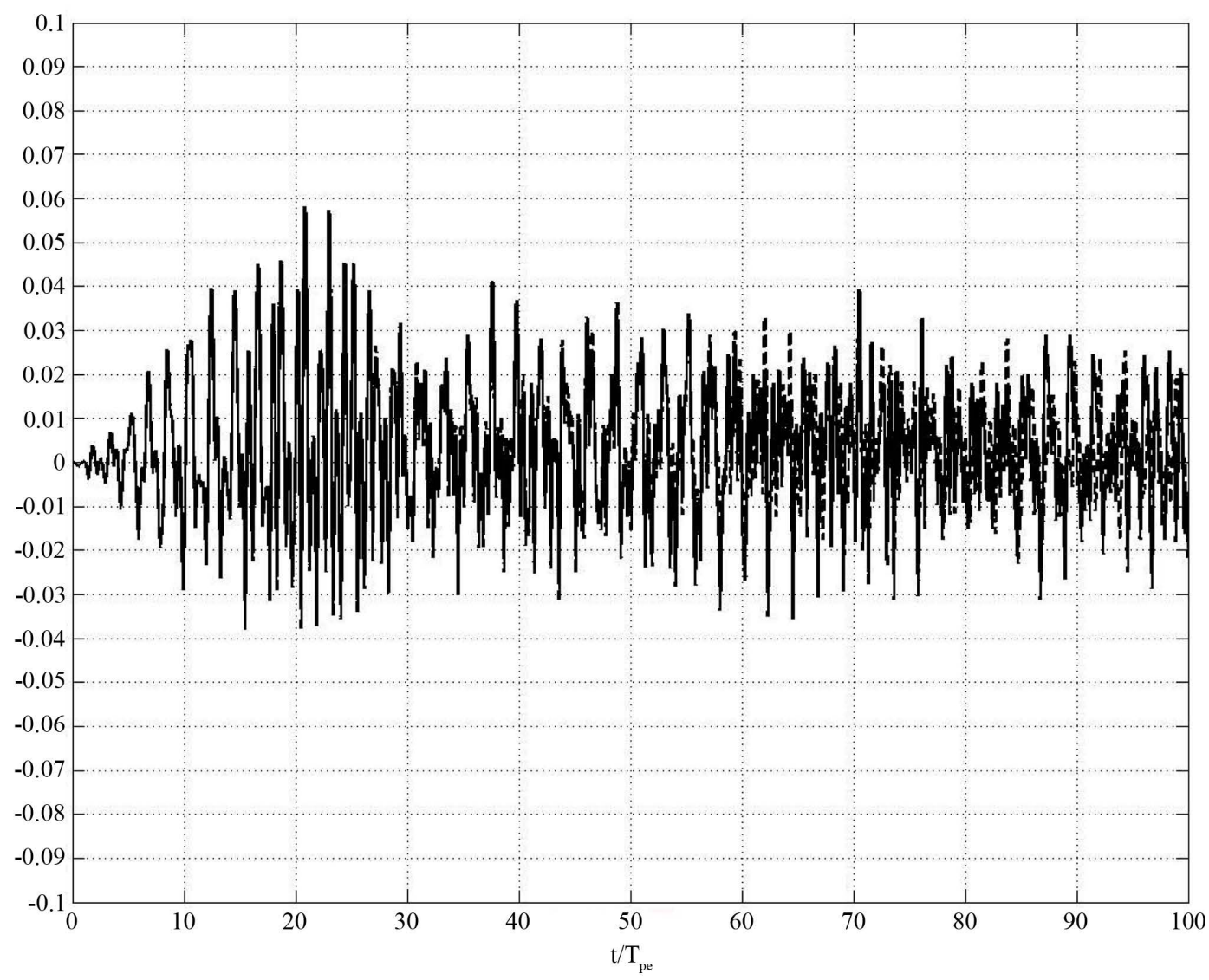

Figure 4. The time variations of one component of the electric field, namely $E_{X}$, lying in the plane perpendicular to the magnetic field, calculated at the point, displaced from the center of the simulation region in the $\boldsymbol{X}$ direction for a distance of sixteen Debye lengths $\left(16 \lambda_{D e}^{0}\right)$. For the first case, when positive ions were supposed to be immovable, the results are shown by solid line, whereas, for the second case, when positive ions can move, the results are shown by dashed line. The electric field component is given in $\mathrm{V} / \mathrm{m}$. The normalized time, $t / T_{p e}$, that is, the time in units of the equilibrium period of Langmuir oscillations of electrons, $T_{p e}$, is shown on the abscissa.

taking into account a motion of the positive ions and a new version of the model has been utilized to study a behavior of initially created irregularities. The utilized model is based on a numerical solution of the VlasovPoisson system of equations, with the Vlasov equations describing the distribution functions of charged particles and the Poisson equation governing the self-consistent electric field. The Vlasov equations are numerically solved applying a macroparticle method. The Poisson equation is solved using a finite-difference method. In the new version of the mathematical model, some parameters of the numerical method have been improved.

The dynamics of the magnetic field aligned irregularity, having circular cross sections, was studied, with the initial cross-section diameter having been equal to twelve Debye lengths of the plasma. Inside the irregularity, the electron concentration is equal to the positive ion concentration at the initial moment, with the concentrations of charged particles being disturbed. Nevertheless, the process started from the completely electrically neutral state in all simulation regions. Calculations were made using the input parameters of the model typical for the nocturnal ionospheric plasma at the altitude of $300 \mathrm{~km}$.

Firstly, calculations were performed for the case when positive ions were supposed to be immovable. Simulation results indicated that the initially created irregularity vanishes and recovers periodically, with its parameters fluctuating. The fluctuations possess of two main periods, namely, the period of Langmuir oscillation of electrons and period of cyclotron oscillations of electrons. It turned out that the irregularity exists during all period of calculations (100 periods of Langmuir oscillations of electrons). The obtained results were compared with 


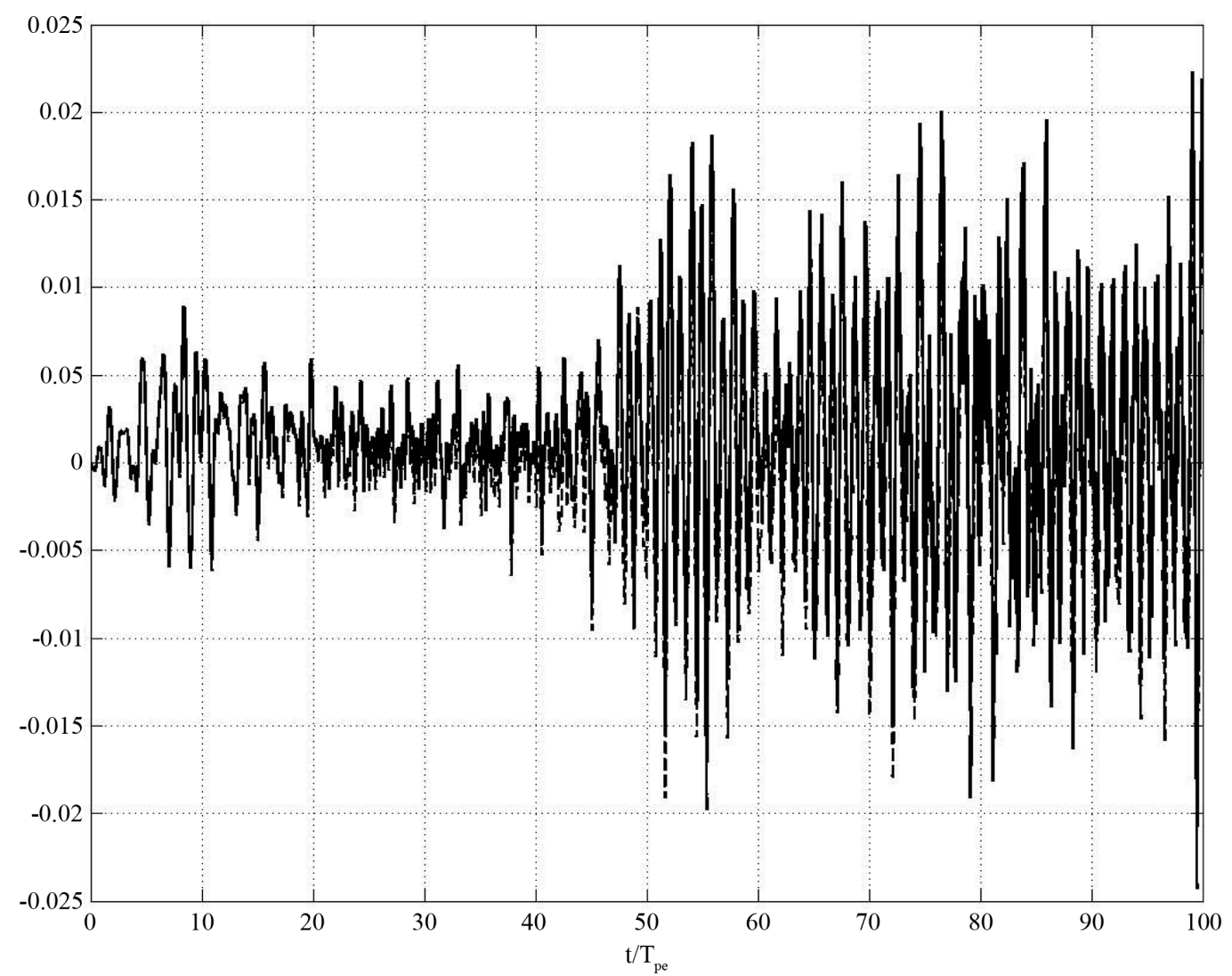

Figure 5. The time variations of one component of the electric field, namely $E_{v}$, lying in the plane perpendicular to the magnetic field, calculated at the point, displaced from the center of the simulation region in the $\boldsymbol{X}$ direction for a distance of sixteen Debye lengths $\left(16 \lambda_{\mathrm{De}}^{0}\right)$. For the first case, when positive ions were supposed to be immovable, the results are shown by solid line, whereas, for the second case, when positive ions can move, the results are shown by dashed line. The electric field component is given in $\mathrm{V} / \mathrm{m}$. The normalized time, $t / T_{p e}$, that is, the time in units of the equilibrium period of Langmuir oscillations of electrons, $T_{p e}$, is shown on the abscissa.

simulation results, obtained in earlier studies devoted to investigation of the dynamics of the irregularity which had not electrical neutrality at the initial moment. For such irregularity, the time interval of about 35 periods of Langmuir oscillations of electrons was sufficient for the irregularity to decay completely. The distinction of the periods of existing may be explained by difference of the distributions of the positive ion concentration inside the irregularities at the initial moment.

Secondly, calculations were performed for the case when the process started from the completely electrically neutral state in all simulation regions and when a motion of the positive ions was taken into account. Obtained simulation results are appeared to be similar to the results obtained for the case when positive ions were supposed to be immovable. Nevertheless, essential distinctions between these simulation results exist. In particular, the behavior of some fluctuating parameters of the plasma, such as X-component of the electric field in the vicinity of the center of the simulation region and the normalized potential energy of the plasma filling up all simulation region, may be different during the initial time interval of about 50 equilibrium periods of Langmuir oscillations of electrons. Thus, the mobility of the positive ions ought to influence essentially on the time evolution of the magnetic field aligned super-small-scale irregularities in the concentration of charged particles, existing in the near-Earth rarefied plasma.

\section{Acknowledgements}

This work was partly supported by Grant No. 13-01-00063 from the Russian Foundation for Basic Research and 


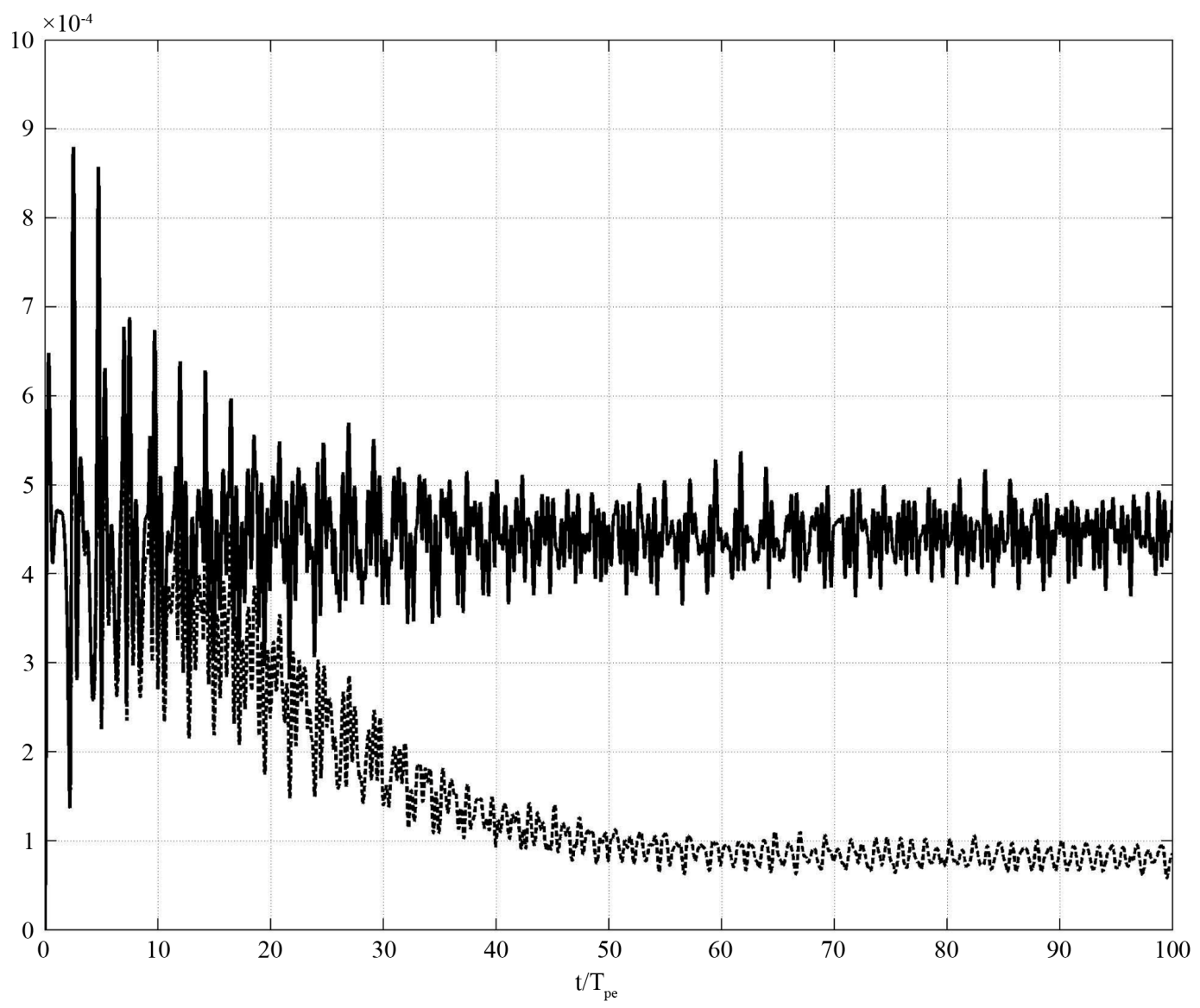

Figure 6. The time variation of the normalized potential energy of the plasma filling up all simulation region, $W_{p o t}(t) / W_{k i n}^{0}$. For the first case, when positive ions were supposed to be immovable, the results are shown by solid line, whereas, for the second case, when positive ions can move, the results are shown by dashed line. The normalized time, $t / T_{p e}$, that is, the time in units of the equilibrium period of Langmuir oscillations of electrons, $T_{p e}$, is shown on the abscissa.

by the Division of the Physical Sciences of the RAS through the program "Plasma processes in space and laboratory".

\section{References}

[1] Ivanov-Kholodny, G.S., Goncharova, E.E., Shashun'kina, V.M. and Yudovich, L.A. (1987) Daily Variations in the Zonal Structure of the Ionospheric F Region at Low Latitudes in February 1980. Geomagnetism and Aeronomia, 27, 722-727.

[2] Moffet, R.J. and Quegan, S. (1983) The Mid-Latitude through in the Electron Concentration of the Ionospheric F-Layer: A Review of Observations and Modelling. Journal of Atmospheric and Terrestrial Physics, 45, 315-343. http://dx.doi.org/10.1016/S0021-9169(83)80038-5

[3] Buchau, J., Reinisch, B.W., Weber, E.T. and Moore, J.G. (1983) Structure and Dynamics of the Winter Polar Cap F Region. Radio Science, 18, 995-1010. http://dx.doi.org/10.1029/RS018i006p00995

[4] Robinson, R.W., Tsunoda, R.T., Vickrey, J.F. and Guerin, L. (1985) Sources of F Region Ionization Enhancement in the Nighttime Auroral Zone. Journal of Geophysical Research, 90, 7533-7546. http://dx.doi.org/10.1029/JA090iA08p07533

[5] Tsunoda, R.T. (1988) High-Latitude F Region Irregularities: A Review and Synthesis. Review of Geophysics, 26, 719760. http://dx.doi.org/10.1029/RG026i004p00719

[6] Besprozvannaya, A.S., Zherebtsov, G.A., Pirog, O.M. and Shchuka, T.I. (1988) Dynamics of Electron Density in the 
Auroral Zone during the Magnetospheric Substorm on December 22, 1982. Geomagnetism and Aeronomia, 28, 66-70.

[7] Muldrew, D.B. and Vickrey, J.F. (1982) High-Latitude F Region Irregularities Observed Simultaneously with ISIS1 and Chatanika Radar. Journal of Geophysical Research, 87, 8263-8272. http://dx.doi.org/10.1029/JA087iA10p08263

[8] Basu, S., Mac Kenzie, E., Basu, S., Fougere, P.F., Maynard, N.C., Coley, W.R., Hanson, W.B., Winningham, J.D., Sugiura, M. and Hoegy, W.R. (1988) Simultaneous Density and Electric Field Fluctuation Spectra Associated with Velocity Shears in the Auroral Oval. Journal of Geophysical Research, 93, 115-136. http://dx.doi.org/10.1029/JA093iA01p00115

[9] Martin, E. and Aarons, J. (1977) F layer Scintillations and the Aurora. Journal of Geophysical Research, 82, 27172722. http://dx.doi.org/10.1029/JA082i019p02717

[10] Fremouw, E.J., Rino, C.L., Livingston, R.C. and Cousins, M.C. (1977) A Persistent Subauroral Scintillations Enhancement Observed in Alaska. Geophysical Research Letters, 4, 539-542. http://dx.doi.org/10.1029/GL004i011p00539

[11] Kersley, L., Russell, C.D. and Pryse, S.E. (1989) Scintillation and EISCAT Investigations of Gradient-Drift Irregularities in the High Latitude Ionosphere. Journal of Atmospheric and Terrestrial Physics, 51, 241-247. http://dx.doi.org/10.1016/0021-9169(89)90075-5

[12] Pryse, S.E., Kersley, L. and Russell, C.D. (1991) Scintillation near the F Layer Trough over Northern Europe. Radio Science, 26, 1105-1114. http://dx.doi.org/10.1029/91RS00490

[13] Greenwald, R.A. (1974) Diffuse Radar Aurora and the Gradient Drift Instability. Journal of Geophysical Research, 79, 4807-4810. http://dx.doi.org/10.1029/JA079i031p04807

[14] Dimant, Ya.S., Oppenheim, M.M. and Milikh, G.M. (2009) Meteor Plasma Trails: Effects of External Electric Field. Annales Geophysicae, 27, 279-296. http://dx.doi.org/10.5194/angeo-27-279-2009

[15] Livingston, R.C., Rino, C.L., Owen, J. and Tsunoda, R.T. (1982) The Anisotropy of High Latitude Nighttime F Region Irregularities. Journal of Geophysical Research, 87, 10519-10526. http://dx.doi.org/10.1029/JA087iA12p10519

[16] Meltz, G. and LeLevier, R.E. (1970) Heating the F-Region by Deviative Absorption of Radio Waves. Journal of Geophysical Research, 75, 6406-6416. http://dx.doi.org/10.1029/JA075i031p06406

[17] Perkins, F.W. and Roble, R.G. (1978) Ionospheric Heating by Radio Waves: Predictions for Arecibo and the Satellite Power Station. Journal of Geophysical Research, 83, 1611-1624. http://dx.doi.org/10.1029/JA083iA04p01611

[18] Mantas, G.P., Carlson, H.C. and La Hoz, C.H. (1981) Thermal Response of F-Region Ionosphere in Artificial Modification Experiments by HF Radio Waves. Journal of Geophysical Research, 86, 561-574. http://dx.doi.org/10.1029/JA086iA02p00561

[19] Bernhardt, P.A. and Duncan, L.M. (1982) The Feedback-Diffraction Theory of Ionospheric Heating. Journal of Atmospheric and Terrestrial Physics, 44, 1061-1074. http://dx.doi.org/10.1016/0021-9169(82)90018-6

[20] Hansen, J.D., Morales, G.J. and Maggs, J.E. (1989) Daytime Saturation of Thermal Cavitons. Journal of Geophysical Research, 94, 6833-6840. http://dx.doi.org/10.1029/JA094iA06p06833

[21] Vas’kov, V.V., Dimant, Ya.S. and Ryabova, N.A. (1993) Magnetospheric Plasma Thermal Perturbations Induced by Resonant Heating of the Ionospheric F-Region by High-Power Radio Wave. Advances in Space Research, 13, 25-33. http://dx.doi.org/10.1016/0273-1177(93)90047-F

[22] Mingaleva, G.I. and Mingalev, V.S. (1997) Response of the Convecting High-Latitude F Layer to a Powerful HF Wave. Annales Geophysicae, 15, 1291-1300. http://dx.doi.org/10.1007/s00585-997-1291-8

[23] Mingaleva, G.I. and Mingalev, V.S. (2002) Modeling the Modification of the Nighttime High-Latitude F-Region by Powerful HF Radio Waves. Cosmic Research, 40, 55-61. http://dx.doi.org/10.1023/A:1014299902287

[24] Mingaleva, G.I. and Mingalev, V.S. (2003) Simulation of the Modification of the Nocturnal High-Latitude F Layer by Powerful HF Radio Waves. Geomagnetism and Aeronomy, 43, 816-825.

[25] Mingaleva, G.I., Mingalev, V.S. and Mingalev, I.V. (2003) Simulation Study of the High-Latitude F-Layer Modification by Powerful HF Waves with Different Frequencies for Autumn Conditions. Annales Geophysicae, 21, 1827-1838. http://dx.doi.org/10.5194/angeo-21-1827-2003

[26] Mingaleva, G.I., Mingalev, V.S. and Mingalev, I.V. (2009) Model Simulation of the Large-Scale High-Latitude FLayer Modification by Powerful HF Waves with Different Modulation. Journal of Atmospheric and Solar-Terrestrial Physics, 71, 559-568. http://dx.doi.org/10.1016/j.jastp.2008.11.007

[27] Mingaleva, G.I., Mingalev, V.S. and Mingalev, O.V. (2012) Simulation Study of the Large-Scale Modification of the Mid-Latitude F-Layer by HF Radio Waves with Different Powers. Annales Geophysicae, 30, 1213-1222. http://dx.doi.org/10.5194/angeo-30-1213-2012

[28] Mingaleva, G.I. and Mingalev, V.S. (2013) Simulation Study of the Modification of the High-Latitude Ionosphere by Powerful High-Frequency Radio Waves. Journal of Computations \& Modelling, 3, 287-309. 
[29] Mingaleva, G.I. and Mingalev, V.S. (2014) Model Simulation of Artificial Heating of the Daytime High-Latitude FRegion Ionosphere by Powerful High-Frequency Radio Waves. International Journal of Geosciences, 5, 363-374.

[30] Eliasson, B. and Stenflo, L. (2008) Full-Scale Simulation Study of the Initial Stage of Ionospheric Turbulence. Journal of Geophysical Research, 113, Article ID: A02305. http://dx.doi.org/10.1029/2007JA012837

[31] Mingalev, O.V., Mingalev, I.V. and Mingalev, V.S. (2006) Two-Dimensional Numerical Simulation of Dynamics of Small-Scale Irregularities in the Near-Earth Plasma. Cosmic Research, 44, 398-408. http://dx.doi.org/10.1134/S0010952506050030

[32] Mingalev, O.V., Mingaleva, G.I., Melnik, M.N. and Mingalev, V.S. (2010) Numerical Modeling of the Behavior of Super-Small-Scale Irregularities in the Ionospheric F2 Layer. Geomagnetism and Aeronomy, 50, 643-654. http://dx.doi.org/10.1134/S0016793210050117

[33] Mingalev, O.V., Mingaleva, G.I., Melnik, M.N. and Mingalev, V.S. (2011) Numerical Simulation of the Time Evolution of Small-Scale Irregularities in the F-Layer Ionospheric Plasma. International Journal of Geophysics, 2011, Article ID: 353640. http://dx.doi.org/10.1155/2011/353640

[34] Wong, A.Y., Santoru, J., Darrow, C., Wang, L. and Roederer, J.G. (1983) Ionospheric Cavitons and Related Nonlinear Phenomena. Radio Science, 18, 815-830. http://dx.doi.org/10.1029/RS018i006p00815

[35] Hockney, R.W. and Eastwood, J.W. (1981) Computer Simulation Using Particles. McGraw-Hill, New York.

[36] Birdsall, C.K. and Langdon, A.B. (1985) Plasma Physics via Computer Simulation. McGraw-Hill, New York. 
Scientific Research Publishing (SCIRP) is one of the largest Open Access journal publishers. It is currently publishing more than 200 open access, online, peer-reviewed journals covering a wide range of academic disciplines. SCIRP serves the worldwide academic communities and contributes to the progress and application of science with its publication.

Other selected journals from SCIRP are listed as below. Submit your manuscript to us via either submit@scirp.org or Online Submission Portal.
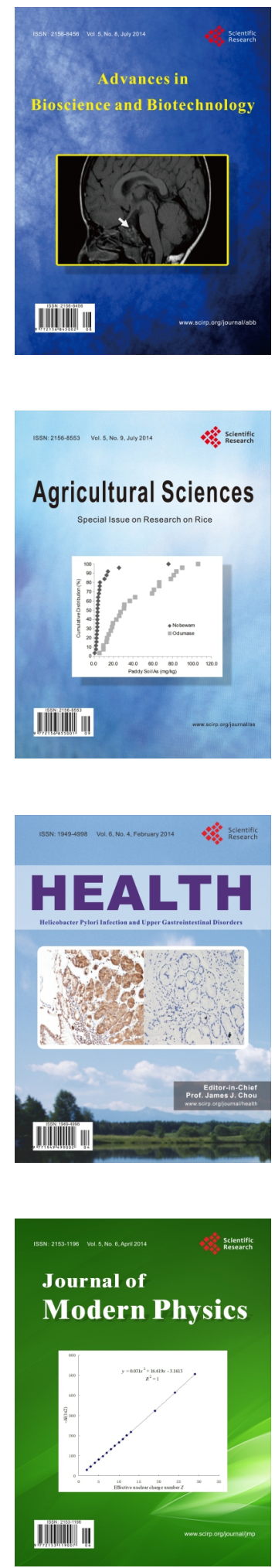
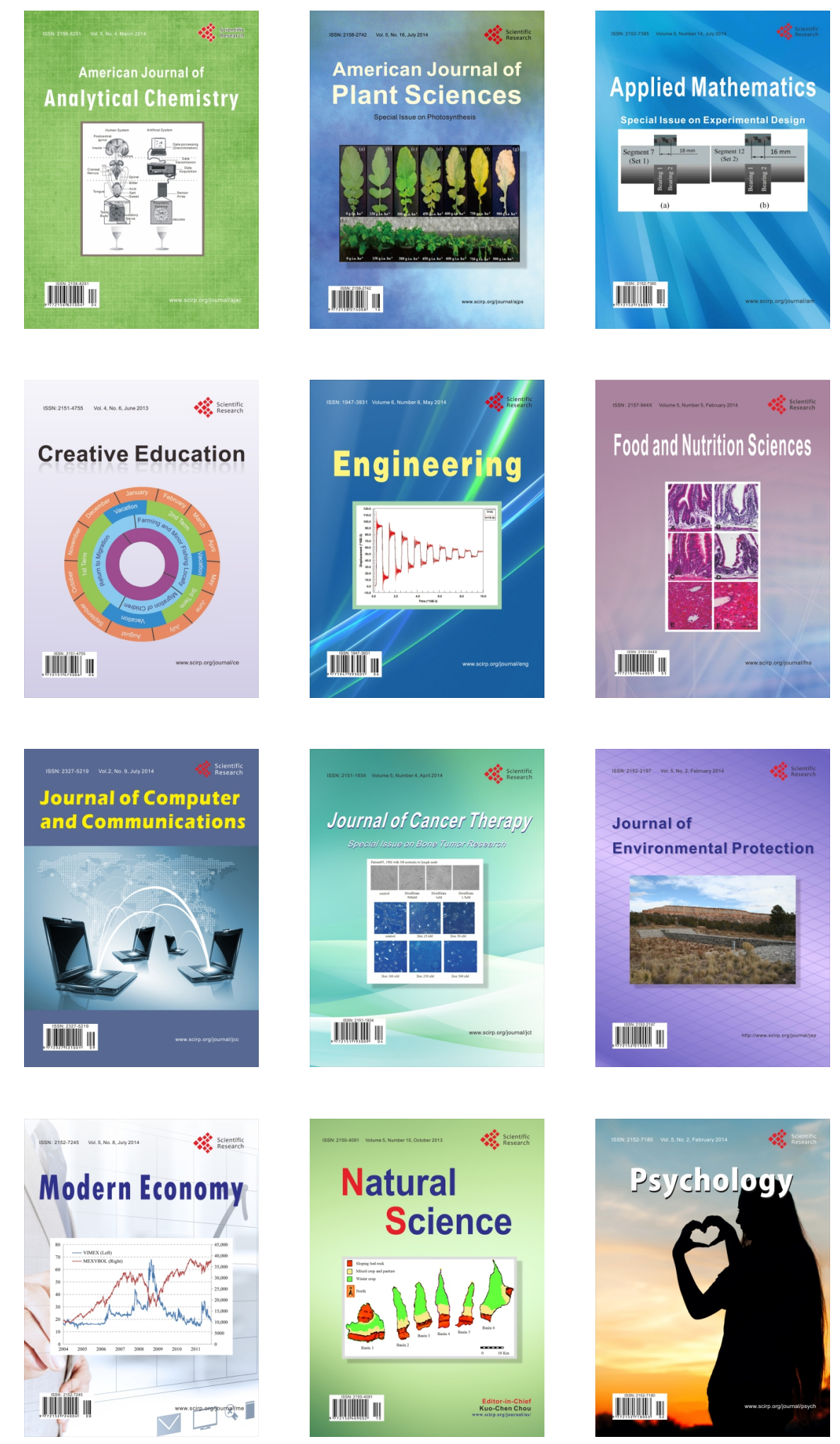